\title{
A NEW RELATION DERIVED FROM PLANCK'S LAW
}

\author{
By Paul D. Foote, Assistant Physicist
}

Of the many possible relations which may be derived from the radiation law of Planck only a few have received any attention. The most important of these are the two displacement laws of Wien; $\lambda_{\max } \theta=$ constant and $J_{\max } \theta^{-5}=$ constant. Salpeter ${ }^{1}$ has defined a function of $\lambda$ and $\theta$ as follows:

$$
\eta(\lambda, \theta)=\frac{J_{\lambda}}{\int_{0}^{\infty} J_{\lambda} d \lambda}
$$

where $J_{\lambda}$ is the intensity of radiation at absolute temperature $\theta$ and wave length $\lambda$ as determined by Planck's law. The functions $\frac{\partial \eta}{\partial \lambda}=o$ and $\frac{\partial \eta}{\partial \theta}=o$ have roots, the former expression giving $\lambda_{m} \theta=$ constant $=2910$ micron degrees for $C_{2}=14450$ micron degrees, i. e., identical with Wien's displacement law, and the latter expression $\lambda \theta_{\max }=3686$ micron degrees for the same value of $C_{2}$.

It is evident that any number of displacement laws may be derived from the Planck equation. ${ }^{2}$ Thus, $\lambda^{\prime} \theta=$ constant where $\lambda^{\prime}$ may refer to any kind of corresponding points on the energy curves at various temperatures, for example, the point of inflection on each side of the maximum ordinate, etc. The present paper concerns the relation that the product of the absolute temperature and the $\lambda$-component of the center of gravity of the spectral energy curve is a constant. The utility of this relation is also briefly considered.

Let

$$
\text { (I) } \begin{aligned}
J_{\lambda}=c_{1} \lambda^{-5}\left(e^{\frac{C_{2}}{\lambda \theta}}-\mathrm{I}\right)^{-1}=\text { Planck's Law } \\
\lambda_{c}=\text { the } \lambda-\text { component of the center of gravity } \\
\text { of the curve } J_{\lambda} \text { versus } \lambda .
\end{aligned}
$$


Then by the definition of center of gravity.

$$
\lambda_{c}=\frac{\int_{0}^{\infty} J_{\lambda} \lambda d \lambda}{\int_{0}^{\infty} J_{\lambda} d \lambda}
$$

Put $x=\frac{C_{2}}{\lambda \theta}$. Equation (2) becomes:

(3)

$$
\lambda_{c}=\frac{C_{2}}{\theta} \frac{\int_{0}^{\infty} x^{2}\left(e^{x}-\mathrm{I}\right)^{-1} d x}{\int_{0}^{\infty} x^{3}\left(e^{x}-\mathrm{I}\right)^{-1} d x}
$$

Expanding $\left(e^{x}-1\right)^{-1}$ by division each term in both numerator and denominator takes the gamma function form, and by the relation:

$$
\int_{0}^{\infty} e^{-k x} x^{n-1}=\frac{\Gamma(n)}{k^{\mathrm{n}}}
$$

equation (3) may be expressed as follows:

$$
\lambda_{c}=\frac{C_{2}}{\theta} \frac{\left(\mathrm{I}+\frac{\mathrm{I}}{2^{3}}+\frac{\mathrm{I}}{3^{3}}+\cdot \cdot \cdot\right)}{\left(\mathrm{I}+\frac{\mathrm{I}}{2^{4}}+\frac{\mathrm{I}}{3^{4}}+\cdot \cdot \cdot\right)} \cdot \frac{\Gamma(3)}{\Gamma(4)}
$$

whence

$$
\text { (.5) } \quad \lambda_{c}=0.3702 \mathrm{x} \frac{C_{2}}{\theta}
$$

Equation (5) is the new relation derived from Planck's law. Thus, for $C_{2}=1445^{\circ}, \lambda_{c} \theta=5350$ micron deg.

The displacement law for the center of gravity holds, of course, for the entire family of curves (I) $\lambda$ versus $J$; (2) $\lambda$ versus $J / \lambda$; (3) $\lambda$ versus $J / \lambda^{2}$, etc. This is illustrated as follows:

\section{Center of gravity $\lambda_{c}$ of} curve from o to $\infty$

(6) $J$ vs. $\lambda$

(7) $J / \lambda$ vs. $\lambda$

(8) $J / \lambda^{2}$ vs. $\lambda$

(9) $J / \lambda^{3}$ vs. $\lambda$

(I0) $J / \lambda^{n}$ vs. $\lambda$

$$
\begin{aligned}
& \lambda_{c}{ }^{(0)}=.3702{ }_{1} C_{2} / \theta \\
& \lambda_{c}{ }^{(1)}=.26094 C_{2} / \theta \\
& \lambda_{c}{ }^{(2)}=.2038{ }_{5} C_{2} / \theta \\
& \lambda_{c}{ }^{(3)}=.{ }^{6} 68 I_{5} C_{2} / \theta
\end{aligned}
$$

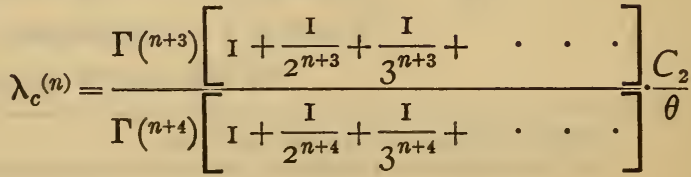


Utility of the Above Relations. - The obvious use to which any of the above equations might be put is the determination of the important constant $C_{2}$ from experimental observation on $J$ and $\lambda$. The observations in general cover a range from $\lambda=.5 \mu$ to $\lambda=6$ to $20 \mu$ while the curves extend to infinity. The center of gravity of a finite portion of the curve (equation (6)) say from $\lambda=0$ to $\lambda=\lambda^{\prime}$ can be determined with a mechanical integrator such as the Amsler integrator No. I. But the center of gravity $\lambda^{\prime}{ }_{c}$ of this portion of the curve will not in general be near enough to the center of gravity $\lambda_{c}$ of the complete energy curve, to permit its use in equations (5) or (6) for the determination of $C_{2}$. The following relation, however, holds for the center of gravity of a finite portion of the curve from $\lambda=0$ to $\lambda=\lambda^{\prime}$ where $p=\frac{C_{2}}{\lambda^{\prime} \theta}$

$$
\lambda^{\prime}{ }_{\mathrm{c}}=\frac{C_{2}}{\theta} \frac{\int_{p}^{\infty} x^{2}\left(e^{x}-\mathrm{I}\right)^{-1} d x}{\int_{p}^{\infty} x^{3}\left(e^{x}-\mathrm{I}\right)^{-1} d x}=\frac{C_{2}}{\theta} f(p)
$$

Equation (II) is readily integrable and a curve of $f(p)$ versus $p$ was constructed. By use of this plot the expression

$$
\lambda^{\prime}{ }_{c}=\frac{C_{2}}{\theta} f\left(\frac{C_{2}}{\lambda^{\prime} \theta}\right)
$$

can be solved for $C_{2}$ by successive approximation. The solution can be computed in less than five minutes. The actual value of this method of obtaining $C_{2}$ depends upon the accuracy with which $\lambda^{\prime}{ }_{c}$ can be determined. Consider a curve extending from o to I I $\mu$ for a temperature of $1800^{\circ}$ abs. The center of gravity of this portion of the curve will lie around $2.8 \mu$. The reading of the integrator is good to about .2 per cent of the total range of wave lengths, II $\mu$, i. e., to $\pm .02 \mu$. Thus $\lambda^{\prime}{ }_{c}$ can be determined to about $\pm \mathrm{I}$ per cent and hence, $C_{2}$ may be computed to this accuracy, neglecting any consideration of errors in $\theta$, etc., since we are considering only mathematical precision. The method accordingly, although probably accurate enough for most radiometric data, and possessing the advantage of being very simple and rapid, does not afford as satisfactory a means of computing $C_{2}$ as the laborious Paschen method or similar methods based on the determination of $\lambda_{\max }$. 
The present brief note was written, not with the primary object of suggesting a new method for the determination of the constant $C_{2}$, but for the purpose of calling attention to a new and interesting displacement law following directly from the Planck spectral distribution equation.

Washington, August I 7, I9I 5 . 